\title{
Characterization of surface electromyography using time domain features for determining hand motion and stages of contraction
}

\begin{abstract}
Surface electromyography (SEMG) signals can provide important information for prosthetic hand control application. In this study, time domain (TD) features were used in extracting information from the SEMG signal in determining hand motions and stages of contraction (start, middle and end). Data were collected from ten healthy subjects. Two muscles, which are flexor carpi ulnaris (FCU) and extensor carpi radialis (ECR) were assessed during three hand motions of wrist flexion (WF), wrist extension (WE) and co-contraction (CC). The SEMG signals were first segmented into $132.5 \mathrm{~ms}$ windows, full wave rectified and filtered with a $6 \mathrm{~Hz}$ low pass Butterworth filter. Five TD features of mean absolute value, variance, root mean square, integrated absolute value and waveform length were used for feature extraction and subsequently patterns were determined. It is concluded that the TD features that were used are able to differentiate hand motions. However, for the stages of contraction determination, although there were patterns observed, it is determined that the stages could not be properly be differentiated due to the variability of signal strengths between subjects.
\end{abstract}

Keyword: Surface electromyography; Time domain features; Prosthetic hand; Stages of contraction; Hand motions 